\title{
A cybernetics approach to inclusive growth analysis: a case of one district
}

\begin{abstract}
Societal transformation towards greater quality of life and well-being requires an inclusive perspective of development. Existing perspectives toward inclusive growth are mainly static. As such, applications of the existing static models are limited as they do not capture the dynamic nature of growth. This problem can be remedied by viewing inclusive growth from a dynamic perspective by means of explaining the dynamic elements of growth and its constraints. Using a cybernetics approach, we illustrate an application of a dynamic inclusive growth analysis of a district. In this study, we define the system-in-focus (inclusive growth in a district) to be viewed from a human activity system. This cybernetics analysis is found to be useful in identifying the growth constraints variables in the district as well as the relationships between them.
\end{abstract}

Keyword: Inclusive growth; Cybernetics analysis; Growth analysis; Qualitative methodology 